\title{
Soliton classical dynamics in the sine-Gordon equation in terms of the massive Thirring model
}

\author{
L. Martinez Alonso \\ Departamento de Métodos Matemáticos de la Física, Facultad de Cíencias Físicas, Universidad Complutense, Madrid-3, Spain
}

(Received 19 July 1984)

\begin{abstract}
The relationship between the soliton dynamics provided by the classical sine-Gordon and massive Thirring models is exhibited. Solitons are characterized as classical relativistic particles through the consideration of their associated canonical realizations of the Poincare group. It is shown that the soliton in the massive Thirring model determines two different kinds of relativistic particles from which sine-Gordon kinks and breathers may be reproduced. In particular, sine-Gordon breathers are characterized as composite systems of two solitons of the massive Thirring model. Soliton scattering in the sine-Gordon equation is described in terms of soliton scattering in the massive Thirring model.
\end{abstract}

\section{INTRODUCTION}

The development of the inverse scattering method (ISM) in the last few years has revealed the existence of a large class ${ }^{1}$ of two-dimensional relativistic field theories which can be solved with the help of an associated spectral problem. It has become clear that most of the interesting properties satisfied by the quantum versions of these models are a consequence of their classical integrability. On the other hand, integrable classical fields, when formulated in the scattering-data language, assume a series of common characteristics inherited from the peculiar structure of the ISM. Thus, they can be understood in terms of two basic components, namely, solitons and radiation, associated with the discrete and continuous scattering data, respectively. The radiation part describes the oscillatory dispersing degrees of freedom, so that solitons are the dominant components in the long time evolution of the field. Since the kinds of solitons as well as the formulas describing their collisions characterize the models solvable by the ISM, one important question is to see to what extent one can establish correspondences among the different integrable relativistic models through their underlying soliton dynamics.

In this paper we shall prove that from the point of view of soliton dynamics there is a close relation between the classical massive Thirring model

$$
\begin{aligned}
\left(i \gamma^{\mu} \partial_{\mu}-m\right) \psi-g^{2}\left(\bar{\psi} \gamma^{\mu} \psi\right) \gamma_{\mu} \psi & =0, \\
& \gamma^{0}=\sigma_{1}, \quad \gamma^{1}=-i \sigma_{2},
\end{aligned}
$$

and the classical sine-Gordon model

$$
\phi_{t t}-\phi_{x x}+\frac{m^{\prime 3}}{\left(g^{\prime}\right)^{1 / 2}} \sin \left(\frac{g^{\prime / 2}}{m^{\prime}} \phi\right)=0,
$$

if the following identifications between the parameters in (1.1) and (1.2) are assumed:

$$
m^{\prime}=m, \quad g^{\prime}=4 m^{2} g^{2} .
$$

As is well known, Coleman proved ${ }^{2}$ that the quantum
sine-Gordon model is equivalent to the charge-zero sector of the quantum massive Thirring model. This important work provided additional incentive for studying these relativistic fields. In particular, a classical version of Coleman's correspondence was proposed by Orfanidis and Wang ${ }^{3}$ before the application of the ISM to the massive Thirring model. ${ }^{4,5}$ However, as was discussed by Kaup and Newell, ${ }^{5}$ this correspondence applies only to singlekink solutions of the sine-Gordon equation and therefore it does not establish any relevant connection between (1.1) and (1.2). In the present paper we consider the soliton dynamics associated with (1.1) and (1.2) as classical pure $S$-matrix theories. Therefore, we are mainly concerned with two aspects: the characterization of solitons as classical relativistic particles and the description of the classical $S$ matrices for soliton scattering. A convenient way to study the particle properties of classical dynamical systems is the use of canonical realizations (CR's) of invariance groups. ${ }^{6}$ In a previous work ${ }^{7}$ we have analyzed the CR of the Poincare group associated with the sineGordon equation with the help of the ISM. As a consequence we characterized the two kinds of solitons arising in the model (kinks and breathers) in terms of simple CR's. In Sec. II of the present paper we perform the analysis of the CR of the Poincare group associated with the invariance of the massive Thirring model under relativistic transformations

$$
\psi^{\prime}\left(t^{\prime}, x^{\prime}\right)=S(\beta) \psi(t, x),
$$

where

$$
S(\beta)=\cosh \frac{\beta}{2}+\gamma^{5} \sinh \frac{\beta}{2}, \quad \gamma^{5}=\gamma^{0} \gamma^{1}=\sigma_{3} .
$$

The use of the action-angle variables corresponding to the completely integrable Hamiltonian structure of $(1.1)^{8}$ makes it possible to express the Poincaré $\mathrm{CR}$ as a direct product of simple CR's. Section III is devoted to the study of the $C R$ associated with the soliton of the massive Thirring model. It is found that the soliton phase space admits lower-dimensional symplectic submanifolds which are invariant under the action of the Poincare group. 
Thus, it follows that the soliton gives rise to two different kinds of relativistic systems: elementary solitons and solitons with a pulsating internal degree of freedom. Section IV deals with the connection between (1.1) and (1.2). Taking into account that two classical relativistic systems can be identified if their respective CR's of the Poincare group are canonically equivalent, our main results may be summarized as follows.

(1) Sine-Gordon kinks are identified with the elementary solitons of the massive Thirring model.

(2) Sine-Gordon breathers are identified with special configurations of composite systems of two pulsating solitons of the massive Thirring model.

(3) In terms of the correspondence defined by (1) and (2), the classical $S$ matrices for soliton scattering in the sine-Gordon and massive Thirring models coincide.

What these results mean is that the classical $S$-matrix theory provided by the soliton dynamics of the sineGordon model can be expressed in terms of the corresponding theory in the massive Thirring model. Since our approach to the relationship between (1.1) and (1.2) is based on the ISM and the Hamiltonian formulation of relativistic invariance, the methods used in this paper may be useful to analyze other integrable relativistic fields.

\section{THE MASSIVE THIRRING MODEL AS A RELATIVISTIC DYNAMICAL SYSTEM}

The invariance of the massive Thirring model under relativistic transformations determines a realization $R$ of the Poincaré group acting on the space of initial data. This realization becomes a canonical one with respect to the symplectic structure generated by the Poisson bracket relations

$$
\begin{aligned}
& \left\{\psi_{\alpha}(x), \psi_{\beta}(y)\right\}=\left\{\psi_{\alpha}^{*}(x), \psi_{\beta}^{*}(y)\right\}=0, \\
& \left\{\psi_{\alpha}(x), \psi_{\beta}^{*}(y)\right\}=-i \delta_{\alpha \beta} \delta(x-y) .
\end{aligned}
$$

The corresponding generators of $R$ are the functionals

$$
\begin{aligned}
P & =\int_{-\infty}^{\infty}\left(-i \bar{\psi} \gamma^{0} \partial_{x} \psi\right) d x \\
H & =\int_{-\infty}^{\infty} h(\bar{\psi}, \psi) d x, \\
K & =\int_{-\infty}^{\infty}\left[-x h(\bar{\psi}, \psi)+\frac{i}{2} \bar{\psi} \gamma^{1} \psi\right) d x,
\end{aligned}
$$

where

$$
h(\bar{\psi}, \psi)=-i \bar{\psi} \gamma^{1} \partial_{x} \psi+m \bar{\psi} \psi+\frac{g^{2}}{2}\left(\bar{\psi} \gamma^{\mu} \psi\right)\left(\bar{\psi} \gamma_{\mu} \psi\right)
$$

They satisfy the Poisson bracket relations

$$
\{P, H\}=0, \quad\{K, H\}=-P, \quad\{K, P\}=-H
$$

which reproduce the Lie-algebra structure of the Poincaré group. It is convenient to use the rescaled field

$$
\phi(t, x)=\left[\frac{2}{m}\right)^{1 / 2} g \psi\left(\frac{2}{m} t, \frac{2}{m} x\right),
$$

which satisfies Eq. (1.1) with $m=2$ and $g^{2}=1$. In terms of $\phi$ the massive Thirring model is equivalent to the commutation equation

$$
\left[X_{0}, X_{1}\right]=0
$$
where $X_{\mu}=X_{\mu}\left(\partial_{\mu}, \lambda, \phi(t, x)\right)$ are the operators introduced
by Mikhailov,

$$
X_{\mu}=2 i \partial_{\mu}+\left(\bar{\phi} \gamma_{\mu} \phi\right) \gamma^{5}+2\left(\begin{array}{cc}
0 & \lambda \phi_{2}^{*}+\epsilon_{\mu} \lambda^{-1} \phi_{1}^{*} \\
\lambda \phi_{2}+\epsilon_{\mu} \lambda^{-1} \phi_{1} & 0
\end{array}\right)-\left(\lambda^{2}+\epsilon_{\mu} \lambda^{-2}\right) \gamma^{5}
$$

where $\left(\epsilon_{0}, \epsilon_{1}\right)=(1,-1)$. The equation $X_{1} g=0$ provides a suitable spectral problem for solving (1.1) through the ISM. In order to introduce the relevant notation about the scattering data, let us consider the Jost solutions $g_{ \pm}(\lambda, x)$ with asymptotic behavior:

$$
\begin{aligned}
& g_{+}(\lambda, x) \underset{x \rightarrow+\infty}{\sim} \exp \left[\frac{i}{2}\left(\lambda^{2}-\lambda^{-2}\right) x\right)\left(\begin{array}{l}
0 \\
1
\end{array}\right), \\
& g_{-}(\lambda, x) \underset{x \rightarrow-\infty}{\sim} \exp \left[-\frac{i}{2}\left(\lambda^{2}-\lambda^{-2}\right) x\right]\left(\begin{array}{l}
1 \\
0
\end{array}\right),
\end{aligned}
$$

and the functions $a(\lambda), b(\lambda)$ verifying

$g_{-}(\lambda, x)=a(\lambda) \hat{g}_{+}(\lambda, x)+b(\lambda) g_{+}(\lambda, x), \operatorname{Im} \lambda^{2}=0$,

where $\hat{g}_{+}(\lambda, x)=\gamma_{1} g_{+}^{*}\left(\lambda^{*}, x\right)$. The function $a(\lambda)$ may be analytically continued to the first and third quadrants of the $\lambda$ plane and its zeros are distributed symmetrically with respect to the origin. We will denote by $\left\{\lambda_{n}\right\}_{n=1}^{N}$ the zeros of $a(\lambda)$ in the first quadrant. Each $\lambda_{n}$ has associat- ed a complex number $c_{n}$ satisfying

$$
g_{-}\left(\lambda_{n}, x\right)=c_{n} g_{+}\left(\lambda_{n}, x\right) \text {. }
$$

The analysis of the spectral transform for the operator $X_{1}=X_{1}\left(\partial_{1}, \lambda, \phi(x)\right)$ shows ${ }^{4,8}$ that $\phi(x)$ is uniquely determined by the set $\left\{\lambda_{n}, c_{n}, b(\lambda) / a(\lambda), b(i \lambda) / a(i \lambda)\right\}$ $(n=1, \ldots, N ; 0<\lambda<\infty)$ of scattering data. It provides an alternative description of the phase space of (1.1).

From the set of scattering data Kuznetsov and Mikhailov $^{8}$ have found the action-angle variables of the massive Thirring model; they are given by

$$
\begin{aligned}
& q_{n}=g^{-2} \ln \left|c_{n}\right|, p_{n}=-4 \ln \left|\lambda_{n}\right|, \\
& \varphi_{n}=\arg c_{n}, \quad \pi_{n}=4 g^{-2} \arg \lambda_{n}, \quad n=1, \ldots, N, \\
& \theta_{1}(k)=\arg b\left(k^{1 / 2}\right), \\
& \rho_{1}(k)=-\left(\pi g^{2} k\right)^{-1} \ln \left|a\left(k^{1 / 2}\right)\right|, \\
& \theta_{2}(k)=\arg b\left(i k^{1 / 2}\right), \\
& \rho_{2}(k)=\left(\pi g^{2} k\right)^{-1} \ln \left|a\left(i k^{1 / 2}\right)\right|, k>0,
\end{aligned}
$$


and the nonzero Poisson bracket relations between them are

$$
\begin{aligned}
& \left\{q_{n}, p_{m}\right\}=\left\{\varphi_{n}, \pi_{m}\right\}=\delta_{n m}, \\
& \left\{\theta_{i}(k), \rho_{j}\left(k^{\prime}\right)\right\}=\delta_{i j} \delta\left(k-k^{\prime}\right) .
\end{aligned}
$$

It must be noticed that according to (2.1) and (2.5) our Poisson bracket definition differs from the one used in Ref. 8 by a factor $-g^{2}$. Through the standard method based on the trace identities for $\ln a(\lambda)$ Kuznetsov and Mikhailov ${ }^{8}$ derived the following expressions for the Poincaré generators $P$ and $H$ in terms of scattering data:

$$
\begin{aligned}
P= & \sum_{n} P_{n}+\int_{0}^{\infty} \frac{m}{2}\left[\frac{1}{k}-k\right]\left[\rho_{1}(k)-\rho_{2}(k)\right] d k \\
H= & \sum_{n}\left(P_{n}{ }^{2}+M_{n}{ }^{2}\right)^{1 / 2} \\
& +\int_{0}^{\infty}\left[\frac{m^{2}}{4}\left[\frac{1}{k}-k\right]^{2}+m^{2}\right]^{1 / 2}\left[\rho_{1}(k)-\rho_{2}(k)\right] d k
\end{aligned}
$$

where

$$
P_{n}=M_{n} \sinh \left(p_{n} / 2\right), \quad M_{n}=2 m g^{-2} \sin \left(g^{2} \pi_{n} / 2\right) \text {. }
$$

We are going to prove that the generator $K$ of pure Lorentz transformations may also be expressed in terms of scattering data as follows:

$$
\begin{aligned}
K= & -2 \sum_{n} q_{n} \\
& +\int_{0}^{\infty}\left[k \rho_{1}(k) \frac{d \theta_{1}(k)}{d k}+k \rho_{2}(k) \frac{d \theta_{2}(k)}{d k}\right] d k .
\end{aligned}
$$

Our derivation of (2.19) is based on the transformation law of the operators (2.7) under pure Lorentz transformations $t^{\prime}=\cosh \beta+x \sinh \beta, x^{\prime}=t \sinh \beta+x \cosh \beta$. It is easy to deduce that

$$
\begin{aligned}
& X_{0}^{\prime}=X_{0} \cosh \beta-X_{1} \sinh \beta, \\
& X_{1}^{\prime}=-X_{0} \sinh \beta+X_{1} \cosh \beta,
\end{aligned}
$$

where $X_{\mu}^{\prime}=X_{\mu}\left(\partial_{\mu}^{\prime}, \lambda^{\prime}, \phi^{\prime}\left(t^{\prime}, x^{\prime}\right)\right)$ and

$$
\begin{aligned}
& \lambda^{\prime}=\exp (-\beta / 2) \lambda, \\
& \phi^{\prime}\left(t^{\prime}, x^{\prime}\right)=S(\beta) \phi(t, x) .
\end{aligned}
$$

Let us consider the Jost solutions $g_{-}(\lambda, t, x)$ and $\hat{g}_{-}(\lambda, t, x)=\gamma_{1} g_{-}^{*}\left(\lambda^{*}, t, x\right)$. They satisfy

$$
\begin{aligned}
& X_{0} g_{-}=-\left(\lambda^{2}+\lambda^{-2}\right) g_{-}, \quad X_{1} g_{-}=0, \\
& X_{0} \hat{g}_{-}=\left(\lambda^{2}+\lambda^{-2}\right) \hat{g}_{-}, \quad X_{1} \hat{g}_{-}=0
\end{aligned}
$$

and form a complete set of solutions of $X_{1} g=0$. From (2.20) and (2.22) we have

$$
\begin{aligned}
& X_{0}^{\prime} g_{-}=-\cosh \beta\left(\lambda^{2}+\lambda^{-2}\right) g_{-}, \\
& X_{1}^{\prime} g_{-}=\sinh \beta\left(\lambda^{2}+\lambda^{-2}\right) g_{-} .
\end{aligned}
$$

These equations together with Eqs. (2.22) and (2.23) imply that the Jost function $g_{-}^{\prime}$ of the operator $X_{1}^{\prime}$ must be of the form

$$
g_{-}^{\prime}\left(\lambda^{\prime}, t^{\prime}, x^{\prime}\right)=c(\lambda, \beta) \exp \left[\frac{1}{2 i} \sinh \left[\left(\lambda^{2}-\lambda^{-2}\right) t^{\prime}-\left(\lambda^{2}+\lambda^{-2}\right) x^{\prime}\right]\right) g_{-}(\lambda, t, x) .
$$

Since $\beta=0$ corresponds to the identity transformation, the coefficient $c(\lambda, \beta)$ in (2.25) verifies $c(\lambda, 0)=1$. Therefore, due to the fact that $g_{-}^{\prime}(\lambda, 0, x)=\exp (\beta K) g_{-}(\lambda, 0, x)$ and $g_{-}(\lambda, t, x)=\exp (t H) g_{-}(\lambda, 0, x)$, Eq. (2.25) implies the following Poisson bracket relation:

$$
\begin{aligned}
\left\{g_{-}(\lambda, x), K\right\}+x & \left\{g_{-}(\lambda, x), H\right\} \\
= & \frac{1}{2} \lambda \partial_{\lambda} g_{-}(\lambda, x)+\frac{i}{2}\left(\lambda^{2}+\lambda^{-2}\right) x g_{-}(\lambda, x) .
\end{aligned}
$$

Hence, taking into account that ${ }^{4,8}$

$$
\{a(\lambda), H\}=0, \quad\{b(\lambda), H\}=i\left(\lambda^{2}+\lambda^{-2}\right) b(\lambda),
$$

by letting $x \rightarrow \infty$ in $(2.22)$, we get

$$
\begin{aligned}
& \{a(\lambda), K\}=\frac{1}{2} \lambda \partial_{\lambda} a(\lambda), \\
& \{b(\lambda), K\}=\frac{1}{2} \lambda \partial_{\lambda} b(\lambda),
\end{aligned}
$$

and this implies

$$
\left\{\lambda_{n}, K\right\}=-\lambda_{n} / 2, \quad\left\{c_{n}, K\right\}=0 .
$$

Thus, the functional $K$ satisfies the following Poisson bracket relations with the action-angle variables $(2.11)-(2.14)$ :

$$
\begin{aligned}
& \left\{q_{n}, K\right\}=\left\{\varphi_{n}, K\right\}=\left\{\pi_{n}, K\right\}=0, \quad\left\{p_{n}, K\right\}=2, \\
& \left\{\theta_{i}(k), K\right\}=k \partial_{k} \theta_{i}(k), \\
& \left\{\rho_{i}(k), K\right\}=\partial_{k}\left[k \rho_{i}(k)\right],
\end{aligned}
$$

which lead at once to (2.19).

Identities (2.16), (2.17), and (2.19) exhibit the decoupling of the contributions of the different pairs of actionangle variables to the Poincaré generators. As a consequence, the CR of the Poincaré group can be represented as a direct product of CR's:

$$
R=\left(\otimes R_{n}\right) \otimes R_{c},
$$

where each factor $R_{n}$ is associated with the variables $\left(q_{n}, P_{n}, \varphi_{n}, \pi_{n}\right)$ and $R_{c}$ is associated with the continuous part of the scattering data. In order to get a simple interpretation of $R_{n}$, it is convenient to introduce three new 
discrete scattering data variables:

$$
\begin{aligned}
& Q_{n}=2 q_{n}\left[M_{n} \cosh \left(p_{n} / 2\right)\right]^{-1}, \\
& \theta_{n}=g^{2} \pi_{n} / 2, \quad \rho_{n}=\frac{2}{g^{2}} \varphi_{n}-\frac{Q_{n} P_{n}}{\tan \theta_{n}} .
\end{aligned}
$$

It is easy to prove that $\left(Q_{n}, P_{n}\right)$ and $\left(\rho_{n}, \theta_{n}\right)$ are pairs of canonically conjugate variables. In terms of them, each CR $R_{n}$ in (2.32) is canonically equivalent to the CR $R_{s}$ of the Poincare group whose phase space $V_{s}$ consists of points $(Q, P, \rho, \theta)$ such that

$$
Q, P \in \mathbb{R}, \rho \in \mathbb{R}\left(\bmod 4 \pi / g^{2}\right), \quad 0<\theta<\pi
$$

and whose generators are

$$
P_{s}=P, \quad H_{s}=\left[P^{2}+M(\theta)^{2}\right]^{1 / 2}, \quad K_{s}=-Q H_{s}
$$

where

$$
M(\theta)=\frac{2 m}{g^{2}} \sin \theta
$$

The factorization (2.32) shows the presence of two kinds of components in the Thirring field. Since discrete scattering data describe soliton degrees of freedom, we have that $R_{s}$ characterizes the soliton as a relativistic dynamical system. On the other hand, as $R_{c}$ is generated by the integral terms in the expressions (2.16) and (2.19), it describes the radiation component of the Thirring field. It is easy to prove that $R_{c}$ is canonically equivalent to the CR of the Poincaré group associated with a free Dirac field of mass $m$.

\section{THE SOLITONS OF THE MASSIVE THIRRING MODEL AS RELATIVISTIC PARTICLES}

In the above section we have found that the soliton of the massive Thirring model is characterized by a CR $R_{s}$ of the Poincare group acting on a phase space with two pairs of canonically conjugate coordinates. Given an element $(b, a, \beta)$ of the Poincare group, from the form (2.36) of $\left\{P_{s}, H_{s}, K_{s}\right\}$ it follows that the points of the phase space transform under $(b, a, \beta)$ according to

$Q^{\prime}=\left(Q H-b P^{\prime}\right) H^{\prime-1}+a, \quad P^{\prime}=H \sinh \beta+P \cosh \beta$,

$\rho^{\prime}=\rho-\frac{1}{2}\left(\frac{2 m}{g^{2}}\right)^{2} \frac{Q \sinh \beta+b}{H^{\prime}} \sin 2 \theta, \quad \theta^{\prime}=\theta$,

where

$$
H=\left[P^{2}+M(\theta)^{2}\right]^{1 / 2}, \quad H^{\prime}=\left[P^{\prime 2}+M(\theta)\right]^{1 / 2} .
$$

On the other hand, the evolution law is given by

$$
\begin{aligned}
& Q(t)=Q(0)+t P / H, \quad P(t)=P(0), \\
& \rho(t)=\rho(0)+\frac{1}{2}\left[\frac{2 m}{g^{2}}\right)^{2} \frac{\sin 2 \theta}{H} t, \quad \theta(t)=\theta(0) .
\end{aligned}
$$

Combining the action of $R_{s}$ with the evolution law, we deduce that the trajectories satisfy the following sample covariant properties:

$$
\begin{aligned}
& Q^{\prime}\left(t^{\prime}\right)=Q(t) \cosh \beta+t \sinh \beta+a, \\
& \rho^{\prime}\left(t^{\prime}\right)=\rho(t),
\end{aligned}
$$

where $t^{\prime}$ is defined by

$$
t^{\prime}=t \cosh \beta+Q(t) \sinh \beta+b .
$$

It is clear that the variables $(Q, P)$ represent the position and the momentum of a classical particle. The remaining variables $(\rho, \theta)$ characterize a pulsating internal degree of freedom whose period in the center-of-mass frame is given by $2 \pi / m \cos \theta$. The best form of illustrating this dynamical system is provided by the field description of the soliton solution which may be written as ${ }^{8}$

$$
\psi_{1,2}^{(s)}(t, x)= \pm\left[\frac{m}{2 g^{2}} \gamma(1 \pm v)\right]^{1 / 2} \sin \theta \frac{\exp [i m \gamma \cos \theta(t-v x)+i \varphi]}{\cosh [m \gamma \sin \theta(x-Q-v t) \pm i \theta / 2]}
$$

where

$$
\gamma=\left(1-v^{2}\right)^{-1 / 2}, \varphi=\frac{g^{2}}{2}\left(\rho+\frac{Q P}{\tan \theta}\right) .
$$

The components of $\psi^{(s)}$ are plane waves modulated by localized bumps whose centers at every instant $t$ are given by $Q(t)$. Observe that the internal variable $\rho$ is related with the motion of the plane wave. The meaning of the variable $\theta$ is particularly interesting. From (2.36), it characterizes the soliton mass, but taking into account the identity $^{8}$

$$
\int_{-\infty}^{\infty} \psi^{\dagger} \psi d x=\sum_{n} 2 g^{-2} \theta_{n}+\int_{0}^{\infty}\left[\rho_{1}(k)+\rho_{2}(k)\right] d k,
$$

we have that $\theta$ is also related to the soliton charge.
From the explicit form (3.8) of the soliton solution one realizes that the value $\theta=\pi / 2$ plays an especially interesting role. Indeed, for this value the internal motion disappears and the soliton behaves as an elementary particle. This fact may be analyzed from the group-theoretical viewpoint as follows. Let us consider the action of the Poincare group on the soliton states. Given an arbitrary real number $\rho_{0}$, Eqs. (3.1) and (3.2) imply that the set of points $(Q, P, \rho, \theta) \in V_{s}$ such that $\rho=\rho_{0}$ and $\theta=\pi / 2$ is invariant under $R_{s}$. These sets are symplectic submanifolds of $V_{s}$ and the corresponding restrictions of $R_{s}$ are canonically equivalent to the elementary CR $R_{e}$ of the Poincare group with phase space $V_{e}=\left\{(Q, P) \in R^{2}\right\}$ and generators

$$
P_{e}=P, \quad H_{e}=\left(P^{2}+M_{e}{ }^{2}\right)^{1 / 2}, \quad K_{e}=-Q H_{e},
$$

where 


$$
M_{e}=\frac{2 m}{g^{2}} .
$$

Thus we have a relativistically invariant characterization of the soliton states with $\theta=\pi / 2$ in terms of elementary particle states.

After picking up the sector $\theta=\pi / 2$ from the soliton phase space, the remaining soliton states appear distributed into two subsets $V_{+}$and $V_{-}$according to the conditions $0<\theta<\pi / 2$ and $\pi / 2<\theta<\pi$, respectively. Clearly $V_{+}$and $V_{-}$are connected symplectic manifolds which are also invariant under $R_{s}$. The corresponding restrictions of $R_{s}$ over $V_{ \pm}$, which characterize the transformation properties of the pulsating soliton states, will be denoted by $R_{P}^{ \pm}$. Their generators have the same form as those of $R_{s}$ given in (2.35). Furthermore, by using the canonical map

$$
V_{+} \rightarrow V_{-}, \quad(Q, P, \rho, \theta) \rightarrow(Q, P,-\rho, \pi-\theta),
$$

it follows that $R_{P}^{+}$is canonically equivalent to $R_{P}^{-}$.

In this way, we conclude that the CR $R_{s}$ admits a reduction which gives rise to two different CR's of the Poincaré group $R_{e}$ and $R_{P}^{ \pm}$describing elementary and pulsating relativistic particles, respectively.

We now turn to the description of the soliton scattering process. As in all wave equations solvable by the ISM, the solitons of the massive Thirring field interact pairwise, so that the whole process may be interpreted as a succession of paired collisions in which every soliton collides with all others. When two solitons collide their action variables remain constant and their angle variables change according to ${ }^{8}$

$$
\begin{aligned}
& \Delta Q_{i}=- \epsilon\left(\beta_{i}-\beta_{j}\right) 2\left(m \sin \theta_{i} \cosh \beta_{i}\right)^{-1} \\
& \times \ln \left|S\left(\beta_{i}-\beta_{j}, \theta_{i}, \theta_{j}\right)\right|, \\
& \Delta \varphi_{i}=\epsilon\left(\beta_{i}-\beta_{j}\right) 2\left[\theta_{j}-\arg S\left(\beta_{i}-\beta_{j}, \theta_{i}, \theta_{j}\right)\right], \quad, j=1,2
\end{aligned}
$$

where

$$
S\left(\beta, \theta_{1}, \theta_{2}\right)=\frac{1-\exp \left[\beta-i\left(\theta_{1}-\theta_{2}\right)\right]}{1-\exp \left[\beta-i\left(\theta_{1}+\theta_{2}\right)\right]},
$$

and where the symbols $\beta_{i}$ denote the rapidities of the solitons

$$
\beta_{i}=\tanh ^{-1} \frac{P_{i}}{H_{i}}=-2 \ln |\lambda i|
$$

and $\epsilon\left(\beta_{i}-\beta_{j}\right)$ is the sign of the relative rapidity. Although we are mainly using the angle variables $\rho_{i}$, Eq. (3.15) refers to the alternative variables $\varphi_{i}$ introduced in (2.12) [see also (2.33) and (3.9)]. Equations (3.14) and (3.15) together with the conservation laws $\Delta P_{i}=\Delta \theta_{i}=0$ specify the classical $S$ matrix for soliton scattering.

\section{THE CONNECTION WITH THE SINE-GORDON EQUATION}

The sine-Gordon model (1.2) has two classes of soliton solutions, namely, structureless solitons (kinks) and pulsating solitons (breathers). The group-theoretical analysis of these solitons, considered as relativistic dynamical systems, was performed in Ref. 7. It was found that the kink solution

$\phi^{(k)}(t, x)=\frac{4 m^{\prime}}{\left(g^{\prime}\right)^{1 / 2}} \tan ^{-1}\left\{\exp \left[ \pm m^{\prime} \gamma(x-Q-v t)\right]\right\}$,

is characterized by a CR $R_{e}^{\prime}$ of the Poincaré group describing an elementary relativistic particle with mass

$$
M_{e}^{\prime}=\frac{8 m^{\prime 3}}{g^{\prime}}
$$

This CR is of the same kind as the CR $R_{e}$ arising in the $\theta=\pi / 2$ soliton sector of the massive Thirring model. In fact, with the identification

$$
\frac{4 m^{\prime 3}}{g^{\prime}}=\frac{m}{g^{2}}
$$

we have that $M_{e}^{\prime}=M_{e}$, and therefore $R_{e}^{\prime}$ and $R_{e}$ are canonically equivalent; that is to say, they describe the same relativistic particle. The breather solution of the sine-Gordon equation

$\phi^{(b)}(t, x)=\frac{4 m^{\prime}}{g^{\prime 1 / 2}} \tan ^{-1}\left[\tan \theta \frac{\sin \left[m^{\prime} \gamma \cos \theta(t-v x)+\varphi\right]}{\cosh \left[m^{\prime} \gamma \sin \theta(x-Q-v t)\right]}\right)$,

is characterized by a CR $R_{b}^{\prime}$ of the Poincare group which acts on a phase space $V_{b}^{\prime}$ with two pairs of canonically conjugate variables $(Q, P, \rho, \theta)$ such that

$Q, P \in \mathbb{R}, \quad \rho \in \mathbb{R}\left[\bmod \frac{32 \pi m^{\prime 2}}{g^{\prime}}\right], 0<\theta<\pi / 2$.

The generators of $R_{b}^{\prime}$ have the form

$$
P_{b}=P, \quad H_{b}=\left[P^{2}+M^{\prime}(\theta)^{2}\right]^{1 / 2}, \quad K_{b}=-Q H_{b},
$$

where the mass function $M^{\prime}(\theta)$ is

$$
M^{\prime}(\theta)=\frac{16 m^{\prime 3}}{g^{\prime}} \sin \theta
$$

In spite of the similarities between $R_{b}^{\prime}$ and the CR $R_{s}$ associated with the soliton of the massive Thirring model, it must be observed that due to the inequality (4.5) for $\theta$ the internal motion is always present in the breather solution. Therefore, we will look for a correspondence between $R_{b}^{\prime}$ and the CR's associated with the pulsating soliton states of the massive Thirring model. These latter have a mass $M(\theta)=M_{e} \sin \theta$, which under the assumption (4.3) becomes half of the breather mass $M^{\prime}(\theta)$. This seems to suggest a description of the sine-Gordon breather as a composite system of two pulsating solitons of the massive Thirring model. Therefore, let us consider the direct product $R_{P}^{+} \otimes R_{P}^{-}$acting on the phase space $V_{+} \times V_{-}=\left\{\left(Q_{i}, P_{i}, \quad \rho_{i}, \theta_{i}\right), \quad i=1,2 ; \quad Q_{i}, P_{i} \in \mathbb{R}^{2}\right.$, $\left.\rho_{i} \in \mathbb{R}\left(\bmod 4 \pi / g^{2}\right), 0<\theta_{1}<\pi / 2, \pi / 2<\theta_{2}<\pi\right\}$, and let $V_{0}$ be the submanifold of $V_{+} \times V_{-}$determined by the constraints

$$
Q_{1}=Q_{2}, \quad P_{1}=P_{2}, \rho_{1}+\rho_{2}=0, \theta_{1}+\theta_{2}=\pi .
$$

It is clear that the restriction of the symplectic structure of $V_{+} \times V_{-}$to $V_{0}$ is nondegenerate and therefore it de- 
fines a symplectic structure on $V_{0}$. We may characterize the points of $V_{0}$ by means of two pairs of canonically conjugate variables $(\hat{Q}, \hat{P}, \hat{\rho}, \hat{\theta})$ defined as

$$
\begin{aligned}
& \hat{Q}=\frac{1}{2}\left(Q_{1}+Q_{2}\right), \hat{P}=P_{1}+P_{2}, \\
& \hat{\rho}=\rho_{1}-\rho_{2}, \hat{\theta}=\theta_{1} .
\end{aligned}
$$

From (3.1) and (4.8) it is elementary to check that $V_{0}$ is invariant under $R_{P}^{+} \otimes R_{P}^{-}$. The generators of the restriction $R_{0}$ of $R_{P}^{+} \otimes R_{P}^{-}$over $V_{0}$ have the same form (4.5) as those of $R_{b}^{\prime}$ with $M^{\prime}(\theta)$ replaced by $2 M(\theta)$. Since (4.3) implies $M^{\prime}(\theta)=2 M(\theta)$, the canonical equivalence between $R_{b}^{\prime}$ and $R_{0}$ will follow if we identify their respective phase spaces. Notice that they differ only in the ranges of variation of the variables $\rho$ and $\hat{\rho}$. However, we can make both coincide by setting

$$
\frac{4 m^{\prime 2}}{g^{\prime}}=\frac{1}{g^{2}} \text {. }
$$

Assumptions (4.3) and (4.10) are equivalent to

$$
m^{\prime}=m, \quad g^{\prime}=4 m^{2} g^{2},
$$

and what we have just proved is that they imply that $R_{b}^{\prime}$ and $R_{0}$ are canonically equivalent.

In this way, under the identification (4.11), the CR's describing kinks and breathers in the sine-Gordon model may be formulated in terms of CR's describing solitons in the massive Thirring model. Thus, as relativistic invariant systems, kinks correspond to $\theta=\pi / 2$ soliton states and breathers correspond to the special configurations (4.8) of clusters consisting of two pulsating solitons. These results have not only a purely kinematical meaning since, as we are going to show, they derive as well from the analysis of the classical $S$ matrices of both models. Let us consider the scattering of two kinks; it is proved in the Appendix that the positions of the interacting kinks become shifted after the interaction according to the formula

$$
\left.\Delta Q_{i}=-\epsilon\left(\beta_{i}-\beta_{j}\right) 2\left(m^{\prime} \cosh \beta_{i}\right)^{-1} \ln |S| \beta_{i}-\beta_{j}, \frac{\pi}{2}, \frac{\pi}{2}\right) \mid
$$

This is exactly the same expression as the one provided by (3.14) for the scattering of two soliton states with $\theta=\pi / 2$ in the Thirring model. Furthermore, as is also proved in the Appendix, the changes of the angle variables in the collision of two breathers are

$$
\begin{aligned}
& \Delta Q_{i}=-\epsilon\left(\beta_{i}-\beta_{j}\right) 2\left(m^{\prime} \sin \theta_{i} \cosh \beta_{i}\right)^{-1}\left[\ln \left|S\left(\beta_{i}-\beta_{j}, \theta_{i}, \theta_{j}\right)\right|+\ln \left|S\left(\beta_{i}-\beta_{j}, \theta_{i}, \pi-\theta_{j}\right)\right|\right], \\
& \Delta \varphi_{i}=-\epsilon\left(\beta_{i}-\beta_{j}\right) 4\left[\arg S\left(\beta_{i}-\beta_{j}, \theta_{i}, \theta_{j}\right)+\arg S\left(\beta_{i}-\beta_{j}, \theta_{i}, \pi-\theta_{j}\right)\right] .
\end{aligned}
$$

From the correspondence established above, the breather variables $(Q, P, \varphi, \theta)$ (see the Appendix) are identified with those $(\hat{Q}, \hat{P}, \hat{\varphi}, \hat{\theta})$ of a two-soliton cluster satisfying (4.8) in the Thirring model, where

$$
\hat{\varphi}=\frac{g^{2}}{2}\left(\hat{\rho}+\frac{\hat{Q} \hat{P}}{\tan \hat{\theta}}\right)=\varphi_{1}-\varphi_{2}=2 \varphi_{1} .
$$

Now, using (3.14) and (3.15) it is elementary to check that (4.13) and (4.15) coincide with the changes $\Delta \hat{Q}_{i}$ and $\Delta \hat{\varphi}_{i}$ arising in the scattering process of two clusters satisfying (4.8), provided the solitons of each cluster are assumed to interact only with the two solitons of the remaining cluster. This confirms the composite structure of the sineGordon breather revealed by the above group-theoretical analysis. Similarly, one may prove that this interpretation is also in accordance with the shift formulas for kinkbreather scattering.

\section{ACKNOWLEDGMENTS}

I am grateful to A. Fernandez Rañada for many helpful discussions and advice. This work was supported in part by the Comision Asesora de Investigación Cientifica $y$ Técnica.

\section{APPENDIX}

The ISM for the sine-Gordon equation is based on the spectral problem ${ }^{9}$

$$
\begin{aligned}
& \left(J \partial_{x}+A+\lambda^{-1} B-\lambda\right) f=0, \\
& J=\left(\begin{array}{cc}
0 & -1 \\
1 & 0
\end{array}\right], \quad A=\frac{i}{4}\left(\begin{array}{cc}
0 & w(x) \\
w(x) & 0
\end{array}\right), \\
& B=\frac{1}{16}\left(\begin{array}{cc}
\exp [i u(x)] & 0 \\
0 & \exp [-i u(x)]
\end{array}\right),
\end{aligned}
$$

where $w \rightarrow 0$ and $u \rightarrow 0(\bmod 2 \pi)$ as $|x| \rightarrow \infty$. Jost solutions are defined through the asymptotic conditions

$$
f_{ \pm}(\lambda, x) \underset{x \rightarrow \pm \infty}{\sim} e_{ \pm}(\lambda, x),
$$

where

$$
e_{ \pm}(\lambda, x)=\exp \left[ \pm i\left(\lambda-16 \lambda^{-1}\right) x\right]\left[\begin{array}{c}
1 \\
\pm i
\end{array}\right)
$$

We introduce two functions $a(\lambda), b(\lambda)$ by means of the asymptotic relation

$$
f_{+}(\lambda, x) \underset{x \rightarrow-\infty}{\sim} a(\lambda) e_{+}(\lambda, x)+b(\lambda) e_{-}(\lambda, x), \quad \lambda \in \mathbb{R} .
$$

The function $a(\lambda)$ may be analytically continued to the half-plane $\operatorname{Im} \lambda>0$. For each zero $\lambda_{n}$ of $a(\lambda)$ a complex number $b_{n}$ exists verifying

$$
f_{+}\left(\lambda_{n}, x\right)=b_{n} f_{-}\left(\lambda_{n}, x\right) \text {. }
$$


The zeros of $a(\lambda)$ are distributed symmetrically with respect to the imaginary axis. Purely imaginary zeros correspond to the presence of kinks in the sine-Gordon field and determine the kink variables in the form ${ }^{7}$

$$
\begin{aligned}
& Q_{n}=-\frac{8}{m^{\prime}}\left(\left|\lambda_{n}\right|^{-1}+16\left|\lambda_{n}\right|\right)^{-1} \ln \left|b_{n}\right|, \\
& P_{n}=\frac{m^{\prime 3}}{g^{\prime}}\left(\left|\lambda_{n}\right|^{-1}-16\left|\lambda_{n}\right|\right) .
\end{aligned}
$$

Observe that in terms of the rapidities $\beta_{n}$ of the kinks

$$
\lambda_{n}=\frac{1}{4} \exp \left(-\beta_{n}+i \frac{\pi}{2}\right)
$$

and

$$
Q_{n}=-\left(m^{\prime} \cosh \beta_{n}\right)^{-1} \ln \left|b_{n}\right| \text {. }
$$

Analogously, the remaining zeros of $a(\lambda)$ are related to the breather variables as follows: ${ }^{7}$

$$
\begin{aligned}
& Q_{n}=-\frac{8}{m^{\prime}}\left[\sin \theta_{n}\left(\left|\lambda_{n}\right|^{-1}+16\left|\lambda_{n}\right|\right)\right]^{-1} \ln \left|b_{n}\right|, \\
& P_{n}=\frac{2 m^{\prime 3}}{g^{\prime}} \sin \theta_{n}\left(\left|\lambda_{n}\right|^{-1}-16\left|\lambda_{n}\right|\right), \\
& \rho_{n}=-\frac{16 m^{\prime 2}}{g^{\prime}} \arg \left(-i b_{n}\right)-\frac{Q_{n} P_{n}}{\tan \theta_{n}}, \theta_{n}=\arg \lambda_{n} .
\end{aligned}
$$

Using the rapidities $\beta_{n}$ of the breathers, these relations imply

$$
\lambda_{n}=\frac{1}{4} \exp \left(-\beta_{n}+i \theta_{n}\right)
$$

and

$$
Q_{n}=-\left(m^{\prime} \sin \theta_{n} \cosh \beta_{n}\right)^{-1} \ln \left|b_{n}\right| .
$$

We define the breather variables $\varphi_{n}$ as

$$
\varphi_{n}=-2 \arg \left(-i b_{n}\right)
$$

which, under the assumptions (4.11) and (A12), may be written as

$$
\varphi_{n}=\frac{g^{2}}{2}\left[\rho_{n}+\frac{Q_{n} P_{n}}{\tan \theta_{n}}\right] .
$$

The sine-Gordon field is completely determined ${ }^{9}$ by the set $\left(\left\{\lambda_{n}\right\}_{n=1}^{N},\left\{b_{n}\right\}_{n=1}^{N}, a(\lambda), b(\lambda)\right)$ of scattering data. Pure soliton solutions are defined by the condition $b(\lambda)=0$.

Let us consider the collision of two single-soliton solutions (kinks or breathers) and let $\left\{\lambda_{1}, b_{1}^{ \pm}, a_{1}(\lambda)\right\}$ and $\left\{\lambda_{2}, b \frac{ \pm}{2}, a_{2}(\lambda)\right\}$ be their scattering data as $t \rightarrow \pm \infty$. Following the procedure due to Manakov ${ }^{10}$ (see also Ref. 8), one finds at once

$$
\frac{b_{1}^{+}}{b_{1}^{-}}=a_{2}\left(\lambda_{1}\right)^{2}, \frac{b_{2}^{+}}{b_{2}^{-}}=a_{1}\left(\lambda_{2}\right)^{-2},
$$

provided $\beta_{1}>\beta_{2}$. The form of the functions $a_{i}(\lambda)(i=1,2)$ depend on the kind of solitons under consideration. Thus, for kinks

$$
a_{i}(\lambda)=\frac{\lambda-\lambda_{i}}{\lambda-\lambda_{i}^{*}}
$$

while, for breathers

$$
a_{i}(\lambda)=\frac{\lambda-\lambda_{i}}{\lambda-\lambda_{i}^{*}} \frac{\lambda+\lambda_{i}^{*}}{\lambda+\lambda_{i}}
$$

From these expressions and using Eqs. (3.16), (A8), and (A13) one deduces for kink-kink scattering

$$
a_{i}\left(\lambda_{j}\right)=S\left[\beta_{j}-\beta_{i}, \frac{\pi}{2}, \frac{\pi}{2}\right]
$$

while, for breather-breather scattering

$$
a_{i}\left(\lambda_{j}\right)=S\left(\beta_{j}-\beta_{i}, \theta_{j}, \theta_{i}\right) S\left(\beta_{j}-\beta_{i}, \theta_{j}, \pi-\theta_{i}\right) .
$$

It is now straightforward to derive from (A9), (A14), (A15), and (A17) the formulas (4.12), (4.13), and (4.14) which describe the soliton shifts for the sine-Gordon equation.
${ }^{1}$ A. V. Mikhailov, Pis'ma Zh. Eksp. Teor Fiz. 30, 443 (1979) [JETP Lett. 30, 414 (1979)]; S. A. Bulgadaev, Phys. Lett. 96B, 151 (1980); O. Babelon, H. J. de Vega, and C. M. Viallet, Nucl. Phys. B190, 542 (1981); F. Lund and T. Regge, Phys. Rev. D 14, 1524 (1976); F. Lund, Phys. Rev. Lett. 38, 1175 (1977); H. J. de Vega and J. M. Maillet, Phys. Rev. D 28, 1441 (1983); A. V. Mikhailov and V. E. Zakharov, Zh. Eksp. Teor. Fiz. 74, 1953 (1978) [Sov. Phys. JETP 47, 1017 (1978)]; H. Eichenherr and M. Forger, Nucl. Phys. B155, 381 (1979); H. J. de Vega, H. Eichenherr, and J. M. Maillet, Commun. Math. Phys. 92, 507 (1984); M. Luscher and K. Pohlmeyer, Nucl. Phys. B137, 46 (1978); H. J. de Vega, Phys. Lett. 87B, 233 (1979).

${ }^{2}$ S. Coleman, Phys. Rev. D 11, 2088 (1975).

${ }^{3}$ S. J. Orfanidis and R. Wang, Phys. Lett. 57B, 281 (1975); S. J. Orfanidis, Phys. Rev. D 14, 472 (1976).
${ }^{4}$ A. V. Mikhailov, Pis'ma Zh. Eksp. Teor. Fiz. 23, 395 (1976) [JETP Lett. 23, 356 (1976)].

${ }^{5}$ D. J. Kaup and A. C. Newell, Lett. Nuovo Cimento 20, 325 (1977).

${ }^{6}$ E. C. G. Sudarshan and N. Mukunda, Classical Dynamics: $A$ Modern Perspective (Wiley, New York, 1974).

${ }^{7}$ L. Martinez Alonso, J. Math. Phys. 24, 982 (1983).

${ }^{8}$ E. A. Kuznetsov and V. Mikhailov, Theor. Math. Phys. 30, 193 (1977).

${ }^{9}$ V. E. Zakharov, L. A. Takhtadzhyan, and L. D. Faddeev, Dok. Akad. Nauk SSSR 214, 1341 (1974) [Sov. Phys. Dok1. 19, 842 (1975)]; L. A. Takhtadzhyan and L. D. Faddeev, Theor. Math. Phys. 21, 1046 (1974).

${ }^{10}$ S. V. Manakov, Zh. Eksp. Teor. Fiz. 67, 543 (1974) [Sov. Phys. JETP 40, 269 (1975)]. 